\title{
Callosal Function in Pediatric Traumatic Brain Injury Linked to Disrupted White Matter Integrity
}

\author{
Emily L. Dennis, ${ }^{1 \star}$ Monica U. Ellis, ${ }^{2,3 *}$ Sarah D. Marion, ${ }^{3}$ Yan Jin, ${ }^{1}$ Lisa Moran, ${ }^{2}$ Alexander Olsen,,${ }^{2,4,5}$ Claudia Kernan, ${ }^{2}$ \\ Talin Babikian, ${ }^{2}$ - Richard Mink, ${ }^{6}$ Christopher Babbitt, ${ }^{7}$ Jeffrey Johnson, ${ }^{8}$ Christopher C. Giza, ${ }^{9}$ \\ Paul M. Thompson, ${ }^{1,10}$ and ${ }^{-R o b e r t ~ F . ~ A s a r n o w ~}{ }^{2,11}$ \\ IImaging Genetics Center, Mary and Mark Stevens Institute for Neuroimaging and Informatics, Keck School of Medicine, University of Southern California, \\ Marina del Rey, California 90292, 2Department of Psychiatry and Biobehavioral Sciences, Semel Institute for Neuroscience and Human Behavior, UCLA, \\ Los Angeles, California 90024, ${ }^{3}$ Fuller Theological Seminary School of Psychology, Pasadena, California 91101, ${ }^{4}$ Department of Psychology, Norwegian \\ University of Science and Technology, NO-7491 Trondheim, Norway, ${ }^{5}$ Department of Physical Medicine and Rehabilitation, St. Olavs Hospital, Trondheim \\ University Hospital, NO-7030 Trondheim, Norway, ${ }^{6} \mathrm{Harbor}-U C L A$ Medical Center and Los Angeles BioMedical Research Institute, Department of \\ Pediatrics, Torrance, California 90502, ${ }^{7}$ Miller Children's Hospital, Long Beach, California 90806, ${ }^{8} \mathrm{LAC}+$ USC Medical Center, Department of Pediatrics, \\ Los Angeles, California 90033, ' UCLA Brain Injury Research Center, Department of Neurosurgery and Division of Pediatric Neurology, Mattel Children's \\ Hospital, Los Angeles, California 90095, ${ }^{10}$ Departments of Neurology, Pediatrics, Psychiatry, Radiology, Engineering, and Ophthalmology, University of \\ Southern California, Los Angeles, California 90033, and ${ }^{11}$ Department of Psychology, UCLA, Los Angeles, California 90024
}

Traumatic brain injury (TBI) often results in traumatic axonal injury and white matter (WM) damage, particularly to the corpus callosum (CC). Damage to the CC can lead to impaired performance on neurocognitive tasks, but there is a high degree of heterogeneity in impairment following TBI. Here we examined the relation between CC microstructure and function in pediatric TBI. We used high angular resolution diffusion-weighted imaging (DWI) to evaluate the structural integrity of the CC in humans following brain injury in a sample of 32 children (23 males and 9 females) with moderate-to-severe TBI (msTBI) at 1-5 months postinjury, compared with well matched healthy control children. We assessed CC function through interhemispheric transfer time (IHTT) as measured using eventrelated potentials (ERPs), and related this to DWI measures of WM integrity. Finally, the relation between DWI and IHTT results was supported by additional results of neurocognitive performance assessed using a single composite performance scale. Half of the msTBI participants (16 participants) had significantly slower IHTTs than the control group. This slow IHTT group demonstrated lower CC integrity (lower fractional anisotropy and higher mean diffusivity) and poorer neurocognitive functioning than both the control group and the msTBI group with normal IHTTs. Lower fractional anisotropy-a common sign of impaired WM-and slower IHTTs also predicted poor neurocognitive function. This study reveals that there is a subset of pediatric msTBI patients during the post-acute phase of injury who have markedly impaired CC functioning and structural integrity that is associated with poor neurocognitive functioning.

Key words: corpus callosum; DTI; ERP; interhemispheric transfer time; traumatic brain injury

Significance Statement

Traumatic brain injury (TBI) is the primary cause of death and disability in children and adolescents. There is considerable heterogeneity in postinjury outcome, which is only partially explained by injury severity. Imaging biomarkers may help explain some of this variance, as diffusion weighted imaging is sensitive to the white matter disruption that is common after injury. The corpus callosum (CC) is one of the most commonly reported areas of disruption. In this multimodal study, we discovered a divergence within our pediatric moderate-to-severe TBI sample 1-5 months postinjury. A subset of the TBI sample showed significant impairment in CC function, which is supported by additional results showing deficits in CC structural integrity. This subset also had poorer neurocognitive functioning. Our research sheds light on postinjury heterogeneity. 


\section{Introduction}

Myelination has a protracted developmental trajectory, continuing well beyond the third decade of life (Bartzokis et al., 2010; Kochunov et al., 2012). In the corpus callosum (CC), fractional anisotropy (FA), a common measure of white matter (WM) integrity-peaks in the mid-30s (Kochunov et al., 2012). WM development supports cognitive development-(Nagy et al., 2004) found a relationship between genu FA and working memory in a pediatric sample. The corpus callosum is the most widely reported area of disruption in traumatic brain injury (TBI; Hulkower et al., 2013). As the largest WM bundle in the brain, the CC is especially vulnerable to traumatic axonal injury (TAI), the progressive axonal disruption often caused by both primary and secondary injuries associated with TBI (Büki and Povlishock, 2006). While only diagnosable definitively postmortem, diffusion-weighted imaging (DWI) provides compelling evidence of TAI in vivo. Across pediatric and adult patients, CC structural integrity is frequently impaired after injury, often leading to functional impairments such as difficulties with bimanual coordination (Caeyenberghs et al., 2011). Deficits in WM integrity associated with TBI can lead to impaired cognitive function, including language and executive function processes, as well as global intelligence measures (Ewing-Cobbs et al., 2008). In children, these deficits can persist years after injury (Ewing-Cobbs et al., 2006).

Interhemispheric transfer time (IHTT) refers to the time required for information to cross cerebral hemispheres via the CC. IHTT has been used to determine CC functional integrity in patients with agenesis of the CC and has shown prolonged CC disruption in case studies of TBI patients (Rugg et al., 1985; Brown et al., 1999; Peru et al., 2003). IHTT, as measured by task reaction time, is impaired after TBI in children (Benavidez et al., 1999) and adults (Mathias et al., 2004). A slower IHTT indicates slower information transfer between the cerebral hemispheres and impaired CC functioning (Brown et al., 1999). Studies of post-TBI hemispheric disconnection (Peru et al., 2003) and surgical sectioning of the splenium (Gazzaniga and Freedman, 1973; Damasio et al., 1980; Sugishita and Yoshioka, 1987) indicate that visual and visuomotor information travels across the splenium.

Here we used event-related potentials (ERPs) to measure IHTT using electroencephalography (EEG) scalp recordings of the posterior brain regions. Prior studies have examined ERP-measured IHTTs in adults with CC damage, but we recently completed the first study examining ERP-measured IHTTs in a sample of pediatric patients with moderate-to-severe TBI (msTBI; Ellis et al., 2015). Here we expand on this prior work by examining the relationship between IHTT and DWI.

Received April 24, 2015; revised May 22, 2015; accepted May 28, 2015.

Author contributions: S.D.M., L.M., A.O., C.K., T.B., R.M., C.B., J.J., C.C.G., P.M.T., and R.F.A. designed research; E.L.D., M.U.E., S.D.M., Y.J., L.M., A.O., C.K., T.B., R.M., C.B., J.J., C.C.G., P.M.T., and R.F.A. performed research;Y.J. and L.M. contributed unpublished reagents/analytic tools; E.L.D., M.U.E., and S.D.M. analyzed data; E.L.D., M.U.E., T.B., P.M.T., and R.F.A. wrote the paper.

This study was supported by the Eunice Kennedy Shriver National Institute of Child Health and Human Development (Grant R01 HD061504). E.L.D., Y.J., and P.M.T. are also supported by the National Institutes of Health Grants U54 EB020403, R01 EB008432, R01 AG040060, and R01 NS080655 to P.M.T., C.C.G. is supported by the UCLA Brain Injury Research Center (Grants NS027544 and NS05489), the Child Neurology Foundation, and the Jonathan Drown Foundation. Scanning was supported by the Staglin IMHRO Center for Cognitive Neuroscience. We thank Alma Martinez and Alma Ramirez for assisting with participant recruitment and study coordination. Finally, we thank the participants and their families for contributing their time to this study.

${ }^{*}$ E.L.D. and M.U.E. contributed equally to this study.

Correspondence should be addressed to Dr. Paul M. Thompson, Keck School of Medicine, University of Southern California, 2001 N. Soto Street, Los Angeles, CA 90033. E-mail: pthomp@usc.edu.

DOI:10.1523/JNEUROSCI.1595-15.2015

Copyright $@ 2015$ the authors $\quad 0270-6474 / 15 / 3510203-10 \$ 15.00 / 0$
Table 1. Demographic information on the participants included

\begin{tabular}{|c|c|c|c|}
\hline & \multicolumn{2}{|l|}{ TBI } & \multirow[b]{2}{*}{$\begin{array}{l}\text { Control group } \\
(N=31)\end{array}$} \\
\hline & $\begin{array}{l}\text { Slow } \\
(N=16)\end{array}$ & $\begin{array}{l}\text { Normal } \\
(N=16)\end{array}$ & \\
\hline Age (years) & $13.9(2.5)$ & $14.5(3.2)$ & $14.9(3.0)$ \\
\hline \multicolumn{4}{|l|}{ Gender } \\
\hline Male & 11 & 12 & 17 \\
\hline Female & 5 & 4 & 14 \\
\hline IHTT (ms) & $25.3(6.2)$ & $7.8(5.2)$ & $9.3(5.4)$ \\
\hline TSI (weeks) & $11.6(4.8)$ & $12.2(5.2)$ & \\
\hline SAH & 4 & 6 & \\
\hline IVH & 2 & 3 & \\
\hline EDH & 7 & 5 & \\
\hline SDH & 6 & 5 & \\
\hline $\mathrm{ICH}$ & 7 & 5 & \\
\hline Contusion & 5 & 5 & \\
\hline DAI & 1 & 3 & \\
\hline $\mathrm{ICP} \uparrow$ & 1 & 4 & \\
\hline \multicolumn{4}{|l|}{ FX } \\
\hline Dep & 5 & 7 & \\
\hline ND & 6 & 3 & \\
\hline
\end{tabular}

Data are average (SD) or $n$. We had acute CT scan information for 15 of 16 IHTT-slow and 15 of 16 IHTT-norma participants. SAH, Subarachnoid hemorrhage; IVH, intraventricular hemorrhage; EDH, epidural hematoma; SDH subdural hematoma; ICH, intracerebral hemorrhage; DAl, diffuse axonal injury; ICP $\uparrow$, increased intracranial pressure; FX, fracture; Dep, depressed; ND, nondepressed.

Table 2. Descriptive statistics on the three IHTT groups

\begin{tabular}{llrlll}
\hline & $N$ & Mean & SD & Minimum & Maximum \\
\hline IHTT-slow & 16 & 25.31 & 6.44 & 18 & 39 \\
IHTT-normal & 16 & 7.81 & 5.35 & 1 & 16.5 \\
Control & 31 & 9.27 & 5.45 & 1.5 & 19 \\
Total & 63 & 12.98 & 9.18 & & \\
\hline
\end{tabular}

Prior studies have examined ERP and DWI data together, but none have examined the data in TBI patients. Multimodal analyses such as these can explain WM disruption in greater depth, revealing mechanisms of impaired function. An earlier study (Babikian et al., 2010) of this cohort used data from DWI, magnetic resonance spectroscopy (MRS), and ERPs, and found a positive association between callosal FA and metabolic markers of neuronal integrity $[N$-acetyl aspartate (NAA)], as well as a negative association between NAA and IHTT. IHTT is related to WM integrity in healthy individuals (Schulte et al., 2005; Westerhausen et al., 2006) and individuals with schizophrenia (Whitford et al., 2011). This study is the first to examine direct associations between WM integrity and IHTT in pediatric TBI patients.

We included 32 pediatric (age range, $8-19$ years) msTBI participants in the post-acute phase (1-5 months postinjury) along with well matched control subjects. Restricting the TBI group to a circumscribed window postinjury is critical because of the dynamic changes in brain structure and function following TBI. We used high-angular resolution diffusion imaging (HARDI), and our novel method for tract extraction, autoMATE (automated multi-atlas tract extraction; Jin et al., 2012; Jin et al., 2013; Jin et al., 2014), which we have shown is sensitive to the disruption caused by TBI (Dennis et al., 2015). We hypothesized that deficits in callosal function, as measured by IHTT, would be associated with deficits in callosal integrity.

\section{Materials and Methods}

\section{Participants}

TBI participants were recruited from four pediatric intensive care units (PICUs) located in level 1 or 2 trauma centers in Los Angeles County. In these institutions, patients with moderate or severe TBI are routinely 
Table 3. Element-wise differences in WM integrity between the IHTT-slow and control groups, and between the IHTT-normal and control groups

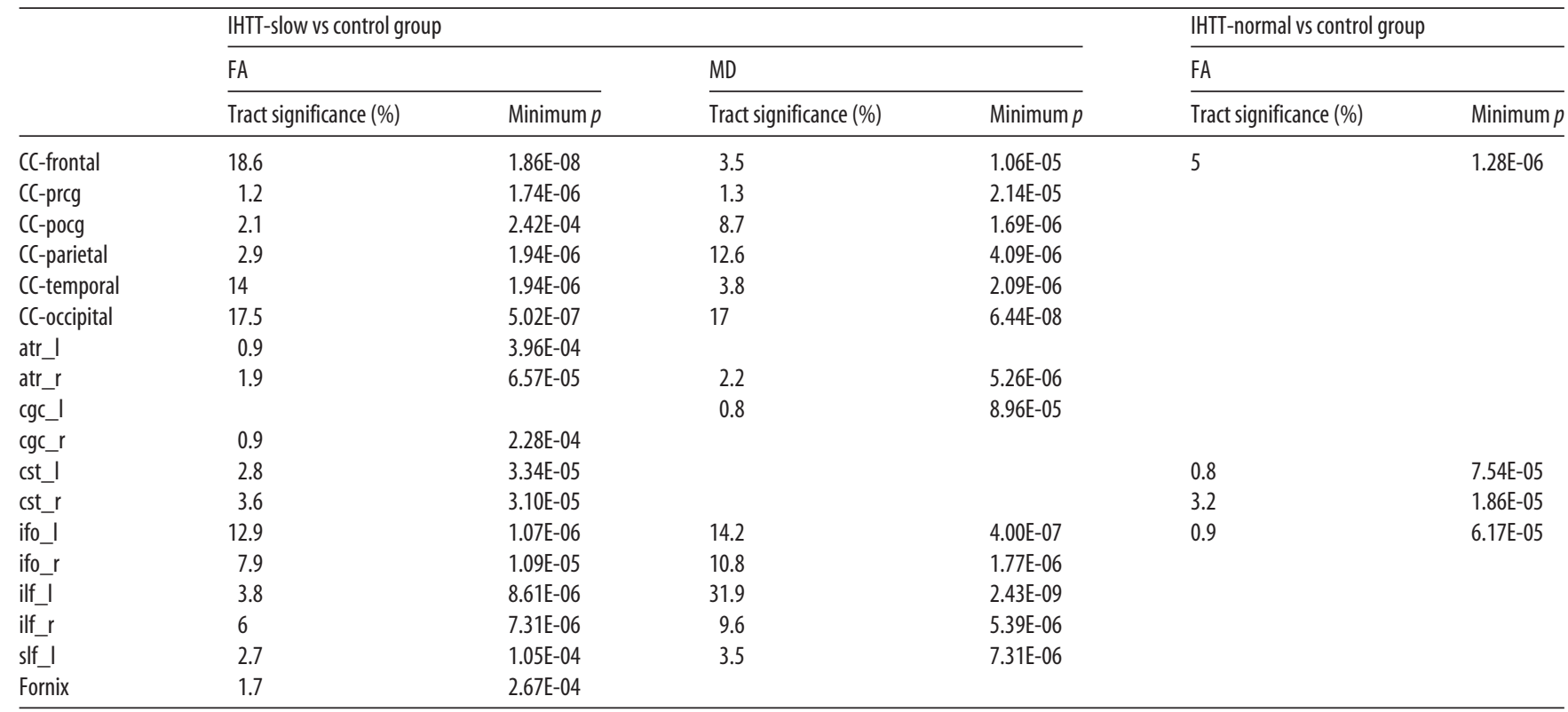

For each tract investigated, the percentage of the tract that passed the FDR threshold is given (FDR threshold for IHTT-slow vs control FA $=0.0038$, for MD $=0.0036$, and for IHTT-normal vs control FA $=6.5 \times 10^{-4}$ ), as well as the minimum $p$ value for group differences on each tract. pocg, Postcentral gyrus; prcg, precentral gyrus.

admitted to the PICU. The study or site coordinator discussed the investigation with the parents of patients, gave them an institutional review board-approved brochure about the study and obtained permission for the investigators to contact them upon discharge. Thirty-five percent of patients whose parents agreed to be contacted while the child was in the PICU participated in this study. Of 124 families contacted at the PICUs, 27 were lost to contact (kept canceling/rescheduling), 21 did not qualify as they did not meet the criteria [e.g., Glasgow Coma Scale (GCS) score of $>12$, English skills not sufficient, attention deficit hyperactivity disorder (ADHD), learning disability, braces], 26 were not interested, 48 are participating, and the participation of 2 is pending. Healthy control subjects, well matched for age, sex, and educational level, were recruited from the community through flyers, magazines, and school postings. We did not have complete ERP and DWI data on all children. We studied 32 children (23 males and 9 females) with msTBI in the post-acute phase (1-5 months postinjury) and 31 healthy control children (17 males and 14 females). Demographics of the participants included in analyses are presented in Table 1. The injury mechanisms for our TBI group were as follows for the total of 32 injured participants: 9 from motor vehicle accident (MVA; pedestrian), 7 from skateboard accident, 6 from MVA (passenger), 2 from scooter accident, 2 from bike accident, 1 from blunt head trauma from sports, 1 from assault, 1 from skiing, 1 from fall from a ladder, 1 from uncategorized blunt head trauma, and 1 from uncategorized skull fracture. From the computed tomography (CT) scan that participants received at the hospital, the prevalence of CT findings was as follows across the 30 participants for whom we had clinical CT scan findings: increased intracranial pressure, 17\%; diffuse axonal injury, $13 \%$; subarachnoid hemorrhage, $30 \%$; ventricular hemorrhage, $17 \%$; epidural hematoma, $40 \%$; subdural hematoma, $37 \%$; intracerebral hematoma, $40 \%$; contusions, $30 \%$; and skull fracture (any, 70\%; depressed skull fracture, 40\%; nondepressed skull fracture, $30 \%)$.

Inclusion criteria. The study inclusion criteria were as follows: (1) nonpenetrating msTBI (intake or post-resuscitation GCS score between 3 and 12); (2) age range, 8-19 years; (3) right handedness; (4) normal visual acuity or vision corrected with contact lenses/eyeglasses; and (5) English skills that were sufficient to understand instructions and be familiar with common words (the neuropsychological tests used in this study presume competence in English).

Exclusion criteria. The study exclusion criteria were as follows: (1) history of neurological illness, such as prior msTBI, brain tumor, or severe seizures; (2) motor deficits that prevent the subject from being examined in an MRI scanner (e.g., spasms); and (3) a history of psychosis, ADHD, Tourette's Disorder, learning disability, mental retardation, autism, or substance abuse. These conditions were identified by parental report and are associated with cognitive impairments that might overlap with those caused by TBI. Participants were excluded if they had metal implants that prevented them from safely undergoing an MRI scan.

\section{Cognitive performance}

Our cognitive performance score is a summary measure assessing multiple domains known to be affected in TBI (Babikian and Asarnow, 2009). It is a linear, unit-weighted combination of the following agebased standardized or scaled measures: (1) Processing Speed Index from the Wechsler Intelligence Scale for Children, fourth edition, (WISC-IV)/ Wechsler Adult Intelligence Scale, third edition (WAIS-III; Wechsler, 2003); (2) Working Memory Index from the WISC-IV/WAIS-III (Wechsler, 2003); (3) Trials 1-5 from the California Verbal Learning Test-Children's Version, second edition (Delis et al., 1994); and (4) Trial 4 from the Delis-Kaplan Executive Function System (Delis et al., 2001).

\section{Scan acquisition}

Participants were scanned on $3 \mathrm{~T}$ Siemens Trio MRI scanners with whole-brain anatomical and a 66-gradient HARDI. DWIs were acquired with the following acquisition parameters: GRAPPA mode; acceleration factor $\mathrm{PE}=2$; TR $9500 \mathrm{~ms} ; \mathrm{TE}=87 \mathrm{~ms} ; \mathrm{FOV}=256 \times 256 \mathrm{~mm}$; isotropic voxel size $=2 \mathrm{~mm}$. Sixty-six images were collected per subject: two $b_{0}$ and 64 diffusion weighted $\left(b=1000 \mathrm{~s} / \mathrm{mm}^{2}\right)$.

\section{Scan comparison}

Part of the way through the study, scanning moved from the UCLA Brain Mapping Center (BMC) to the Staglin IMHRO Center for Cognitive Neuroscience (Staglin). Both scanners were 3 T Siemens Trio scanners, and the protocol was maintained. To determine whether this scanner change introduced bias into our data, we scanned six healthy adult volunteers at both the BMC and Staglin, 1.5 months apart. We then assessed the possible bias in both of the T1-weighted images. Extensive details on this process may be found in our study (Dennis et al., 2015). For the T1-weighted images, the scan comparison analyses revealed no detectable pattern in the difference between the intensity correction fields above noise, except in the cerebellum, where there were some scannerinduced differences in image intensity even after N3 correction (intensity correction). For this reason, the cerebellum was masked from the analyses presented in this article. 


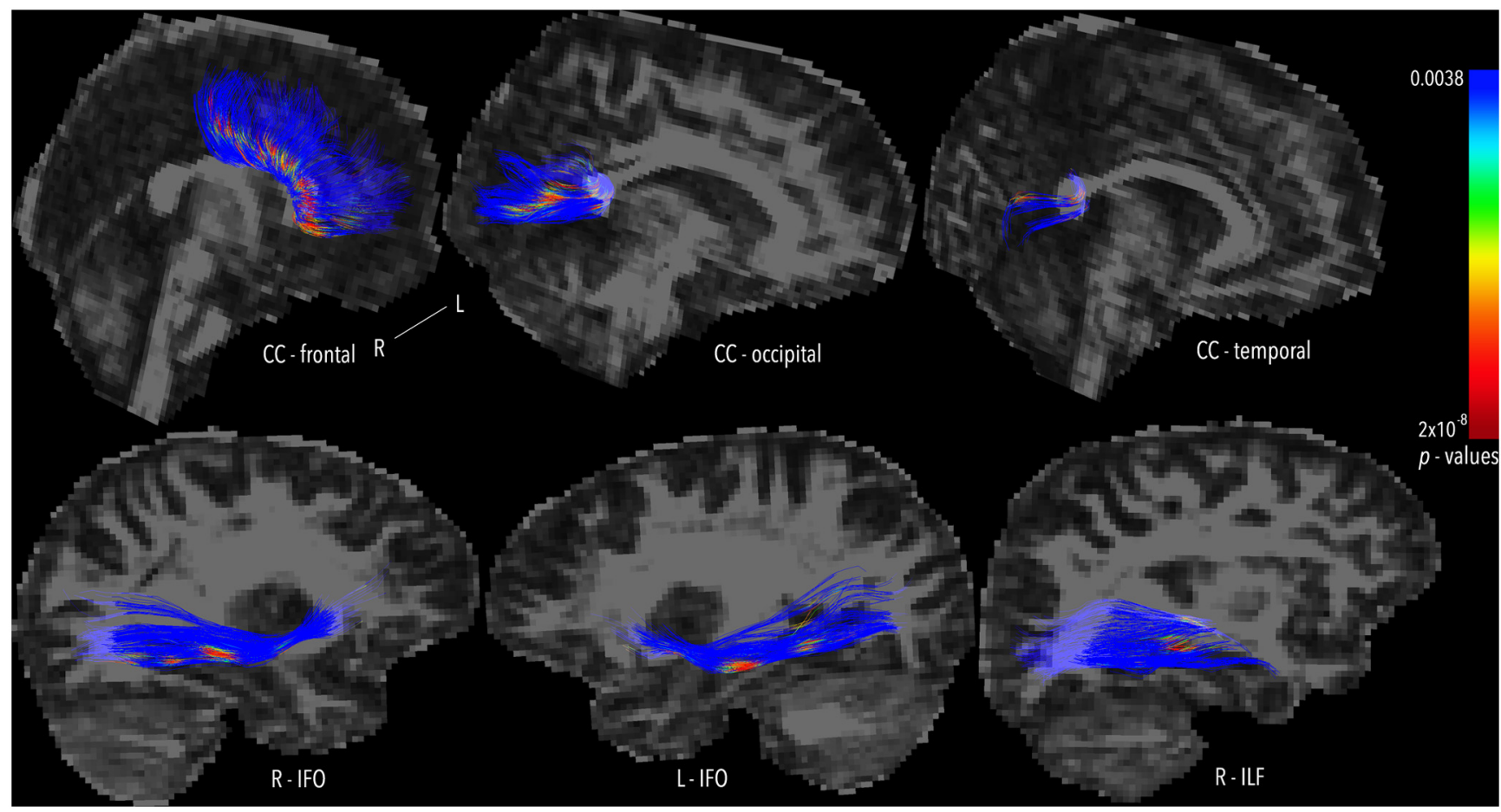

Figure 1. Differences between IHTT-slow and control groups in FA. Whole-tract differences in FA between IHTT-slow and control. The $p$ values are shown corresponding to the color bar, and results were FDR corrected across all points on all tracts tested $(q<0.05$; critical $p$ threshold $=0.0038$ ). For simplicity, only tracts with at least $5 \%$ of the tract showing significant differences are displayed. IF0, Inferior fronto-occipital fasciculus; ILF, inferior longitudinal fasciculus.

\section{Tractography}

autoMATE, developed by our laboratory, has been described fully in prior articles (Jin et al., 2012, 2013, 2014). Raw HARDI images were visually checked for artifacts, resulting in two participants being excluded from all analyses due to extensive slice dropout (not included in the above participant count). HARDI images were corrected for eddy current-induced distortions using the FSL tool "eddy_correct" (http:// fsl.fmrib.ox.ac.uk/fsl/). DWI scans were skull stripped using "BET." FA and mean diffusivity (MD; also called apparent diffusion coefficient) maps were computed using "dtifit." Whole-brain HARDI tractography was performed with Camino (http://cmic.cs.ucl.ac.uk/camino/). The maximum fiber turning angle was set to $35 \%$ voxel to limit biologically implausible results, and tracing stopped when FA dropped $<0.2$, as is the standard in the field.

\section{Fiber clustering and label fusion}

As part of autoMATE, five WM tract atlases were constructed from the HARDI data of healthy young adults (20-30 years old), as detailed previously (Jin et al., 2012, 2013, 2014). The atlas, based on the "Eve" brain atlas (Zhang et al., 2010), includes the following 18 major WM tracts: the anterior thalamic radiation [left (atr_l) and right (atr_r)], corticospinal tract [left (cst_l) and right (cst_r)], cingulum [left (cgc_l) and right (cgc_r)], inferior fronto-occipital fasciculus [left (ifo_l) and right (ifo_r)], inferior longitudinal fasciculus (left (ilf_l) and right (ilf_r)], arcuate fasciculus [left (arc_l; right arcuate is too asymmetric for population studies to be practical; Catani et al., 2007)], fornix, and corpus callosal tracts divided into six segments (frontal, precentral gyrus, postcentral gyrus, parietal, temporal, and occipital). The Eve atlas was registered, linearly and then nonlinearly, to each subject's FA map using Advanced Normalization Tools (Avants et al., 2011), and its ROIs were correspondingly warped to extract 18 tracts of interest for each subject based on a lookup table (Zhang et al., 2010). ROI registration was visually checked for all subjects, and all passed quality control. Each subject's FA map was further registered nonlinearly to each of the five manually constructed atlases. All registrations were visually inspected for quality, and all passed quality control. The 18 tracts from each atlas were then warped to the subject space based on the deformation field from the registration steps (Jin et al., 2011). We refined fiber extractions of each tract based on the distance between the warped corresponding tract of each atlas and the subject's fiber candidates from ROI extraction. Individual results from the five atlases were fused. We visually inspected the resulting fiber bundles. For each of the $18 \mathrm{WM}$ tracts, we selected one example subject to display the results of group analyses. FA is the degree to which water is diffusing preferentially in one direction (along axons). MD is a related measure of the average diffusivity across all three primary eigenvectors and typically increases when FA decreases. We extracted tract-based FA and MD data at this point for group comparisons.

There may be some concern that the age range ( $8-19$ years) might have an impact on DWI postprocessing, in terms of different accuracy of registration or warping of scans to the template, with respect to age. To mitigate this, we used our multitemplate approach for tract extraction that allows for the fibers and pathways to show some allometry; in other words, it does not assume that the relative volumes of the brain regions stay constant with age or that the tracts will always be in the same template coordinates in children of different ages. As we used multiple tract templates, there is some added versatility in the method to "catch" the relevant fibers in each individual even if the nonlinear registration is not perfect and if individual anatomy is different from each of the individual templates.

\section{Visual ERP measure of IHTT}

This study used visual ERPs to measure IHTT. IHTT is defined as the time required to transfer stimulus-locked neural activity between the left and right brain hemispheres in the participants. Electroencephalography was recorded while participants completed a computerized, patternmatching task with bilateral field advantage. Participants were exposed to two distinct visual patterns (geometric shapes of 9-11 letter "o"s) following a gaze fixation (a colon) in the center of the visual field. These patterns were presented randomly to two of the four visual fields (upper and lower; left and right). This created four bilateral and two unilateral conditions [right visual field (RVF) and left visual field (LVF)]. Participants were asked to determine whether the two patterns presented during each trial constituted a "match" or a "nonmatch." Match and nonmatch re- 


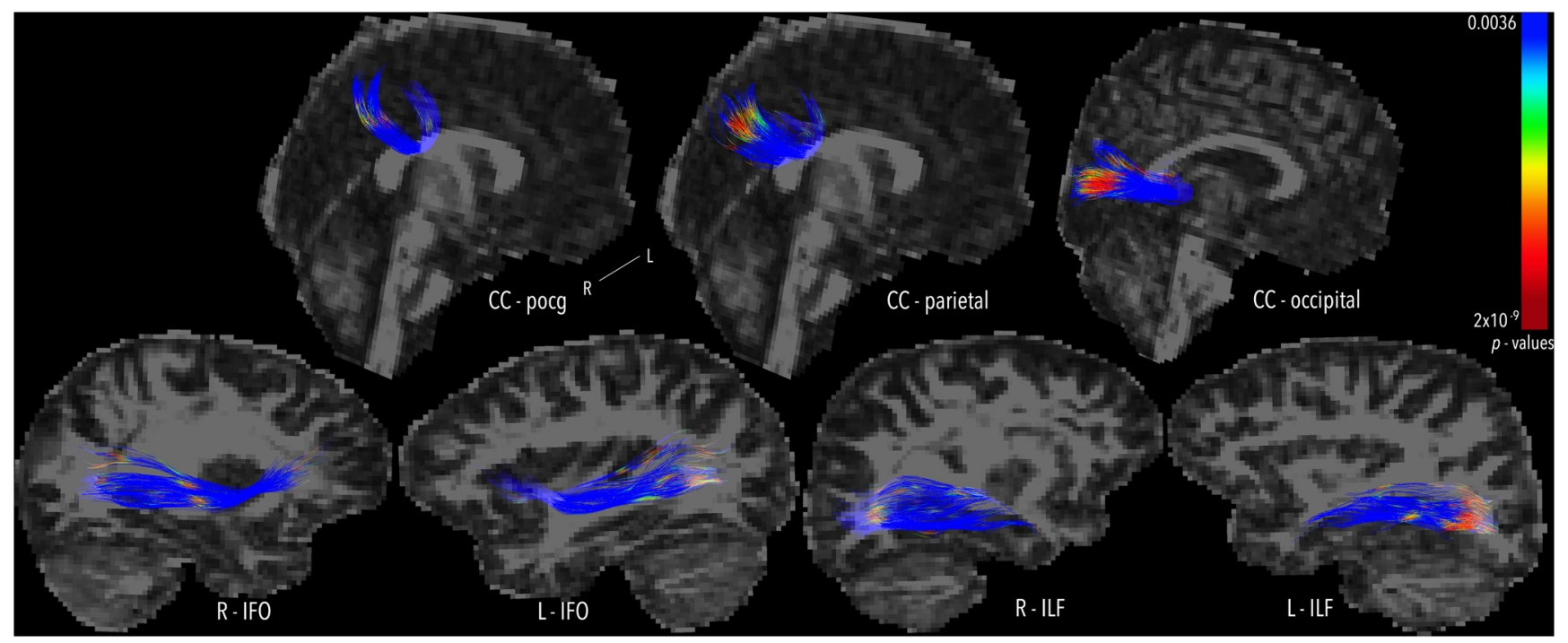

Figure 2. Differences between IHTT-slow and control groups in MD. Whole-tract differences in MD between IHTT-slow and control groups. The $p$ values are shown corresponding to the color bar, and results were FDR corrected across all points on all tracts tested $(q<0.05$; critical $p$ threshold $=0.0036)$. For simplicity, only tracts with at least $5 \%$ of the tract showing significant differences are displayed.

sponses were made by pressing the " $\mathrm{M}$ " and " $\mathrm{N}$ " keys of a computer keyboard. Participants alternated between pressing with the right hand - with their index finger on the $\mathrm{N}$ key and their middle finger on the M key — or with the left hand-with their index finger on the M key and middle finger on the $\mathrm{N}$ key-for each trial. The responding hand was alternated in eight blocks of 97 trials. Each participant's cross-callosal IHTT was calculated using the electroencephalographic visual ERPs, which were collected during the unilateral conditions. Greater detail on this method has been described previously (Larson and Brown, 1997).

\section{ERP recording}

Electroencephalography was recorded while participants completed a computerized, pattern-matching task with bilateral field advantage. A BIOSEMI system was used to acquire ERPs. Visual ERPs were recorded and synchronized to the onset of the pattern presentation (low-pass filter $=40 \mathrm{~Hz}$; high-pass filter $=0.16 \mathrm{~Hz} ; 3 \mathrm{~dB}$ bandwidth $=134 \mathrm{~Hz}$; sample rate $=512 \mathrm{~Hz}$ ). Parietal and occipital electrode sites were used because previous studies have shown that these lateral sites produce large visual ERPs that yield clear evoked potential IHTTs (Andreassi et al., 1975; Ledlow et al., 1978; Brown and Jeeves, 1993). In addition, electrodes were placed above, below, and at the outer cantus of each eye for the recording of eye movements. Electrodes placed on the mastoid bones (i.e., behind the ears) of participants were used as linked-ears references. This reference point provides a more valid estimate of IHTT than midfrontal reference points (Saron and Davidson, 1989). ERPs at each recording electrode from each trial were stored for later averaging.

For each electrode, ERPs were averaged for the $2 \times 2$ combinations of LVF versus RVF. Averaged ERPs were displayed on a computer visual display, and the N1 component was identified with the participant blinded to the participant group. For each of the parietal or occipital recording electrodes, which were contingent upon which set provided the most clearly identifiable nodes and peaks, the latency and amplitude of these components were stored for statistical analysis. IHTT was calculated by averaging ERP waveforms at the $\mathrm{P} 3$ or $\mathrm{O} 1$ (left hemisphere) and $\mathrm{P} 4$ or $\mathrm{O}_{2}$ (right hemisphere) electrode sites. Next, the peak latency (in milliseconds) of the early N1 evoked potential components was determined. Then, the latencies of the ipsilateral and contralateral conditions were subtracted to determine the overall IHTT for each visual field. Finally, the RVF and LVF IHTTs were averaged to compute the overall IHTT for each participant. As mentioned, the average of the left-toright visual field and right-to-left visual field IHTTs for each participant was used in the remaining analyses. Longer IHTTs indicate slower transfer of visual information across the posterior brain regions. Accuracy of pattern matching was not recorded in this study. Prior studies have demonstrated that true deficits in IHTT are related to slower reaction times rather than to response accuracy, particularly in individuals with agenesis of the corpus callosum (Sauerwein and Lassonde, 1983; Lassonde et al., 1988).

\section{Group comparison}

We ran an element-wise linear regression (treating each data point along the tract as a separate element) testing for association between IHTT and FA or $\mathrm{MD}$, including age, sex, scanner, and group (TBI vs control) as covariates. These were corrected for multiple comparisons using the false discovery rate (FDR) method ( $q<0.05$; Benjamini and Hochberg, 1995). We also examined the data in a different way. Given preliminary results suggesting a bimodal distribution of IHTTs in the TBI group, with one subgroup having IHTTs that were not significantly different from those of control subjects, and another group with IHTTs that differed by at least 1.5 SDs, we examined each group separately. We ran element-wise linear regression testing for group differences between the IHTT-slow TBI group and the control subjects, and between the IHTT-normal TBI group and the control subjects, again with age, sex, and scanner as covariates.

\section{Cognitive analysis}

We ran an element-wise linear regression testing for the association between cognitive performance and FA or MD, including age, sex, and scanner as covariates. These were corrected for multiple comparisons using the FDR method ( $q<0.05$; Benjamini and Hochberg, 1995). To examine the joint impact of IHTT and FA on cognitive performance, we ran an element-wise partial $F$ test, comparing the following two models:

$$
\text { Reduced model: Performance Index } \sim \text { age }+ \text { sex }+I H T T_{\text {group }}
$$

Full model: Performance Index $\sim$ age + sex $+I H T T_{\text {group }}$

$$
+F A c_{(X, Y)} \text {. }
$$

Here IHTT $_{\text {group }}$ is a numeric variable with $1=$ IHTT-slow, $2=$ IHTTnormal, and $3=$ control; and $\mathrm{FAc}_{(X, Y)}$ is an element-wise centered FA, where $(X, Y)$ are the matrix coordinates corresponding to tract indices. We iterated through this partial $F$ test for each tract index $(5607 \times 15$ indices in total) to create a $5607 \times 15$ matrix of $F$ statistics. These were FDR corrected for multiple comparisons across all indices on all tracts $(q<0.05)$. The scanner did not have a significant effect on performance index, so, to simplify the model, it was not included. We also ran these analyses including scanner as a covariate in both models, and it did not 


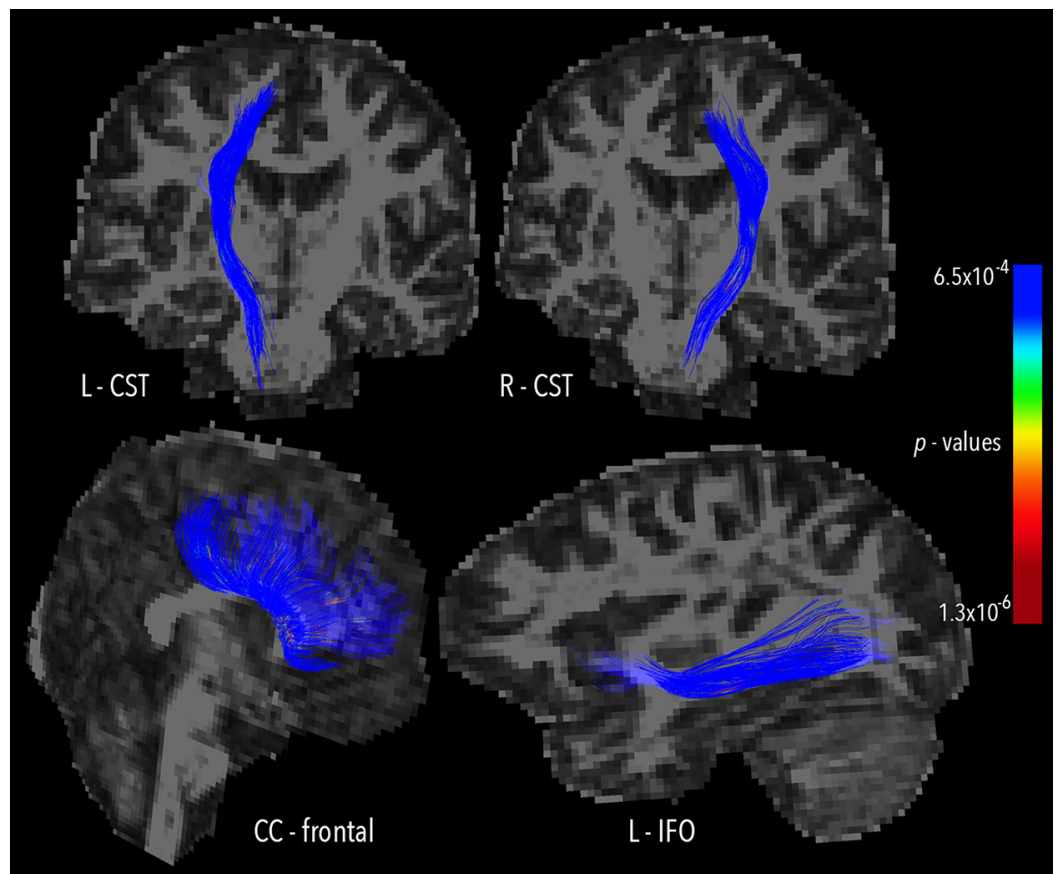

Figure 3. Differences between IHTT-normal and control groups. Whole-tract differences in FA between IHTT-normal and control groups across all tracts tested. The $p$ values are shown corresponding to the color bar, and results were FDR corrected across all points on all tracts tested $\left(q<0.05\right.$; critical $p$ threshold $\left.=6.5 \times 10^{-4}\right)$. All tracts showing significant differences are shown. CST, Corticospinal tract; IF0, inferior fronto-occipital fasciculus.

Table 4. Descriptive statistics on the performance index scores of the three IHTT groups

\begin{tabular}{llrlll}
\hline Performance index & $N$ & \multicolumn{1}{c}{ Mean } & SD & Minimum & Maximum \\
\hline IHTT-slow & 16 & 91.38 & 12.52 & 76.88 & 124.5 \\
IHTT-normal & 16 & 99.88 & 11.04 & 75.75 & 114.88 \\
Control & 31 & 104.08 & 10.75 & 74.25 & 128.5 \\
Total & 63 & 99.78 & 12.28 & & \\
\hline
\end{tabular}

Table 5. The results of our partial $F$ test comparing a reduced model (age, sex, and IHTT group) with a full model (age, sex, IHTT group, and element-wise FA) with cognitive performance as the response variable

\begin{tabular}{lcl}
\hline & Tract significance (\%) & Average Fstatistic \\
\hline CC-frontal & 16.0 & 6.4 \\
CC-parietal & 3.3 & 5.2 \\
CC-pocg & 4.8 & 6.3 \\
CC-prcg & 5.1 & 5.3 \\
CC-temporal & 4.7 & 5.5 \\
CC-occipital & 2.8 & 6.5 \\
atr_I & 9.7 & 7.1 \\
atr_r & 10.0 & 7.0 \\
cgc_I & 4.9 & 5.6 \\
cgc_r & 6.7 & 5.4 \\
cst_I & 2.4 & 5.3 \\
cst_r & 10.5 & 6.1 \\
Fornix & 3.0 & 5.4 \\
ifo_I & 2.9 & 5.4 \\
ifo_r & 12.8 & 6.3 \\
ilf_I & 3.2 & 5.4 \\
ilf_r & 9.1 & 6.8 \\
arc_I & 4.6 & 5.4 \\
\hline
\end{tabular}

For each tract investigated, the percentage of the tract with a significant $F$ statistic is given, along with the average F statistic along the tract (nonzero elements). Results were FDR corrected $(q<0.05)$.

affect the results. We ran our partial $F$ test according to the following formula:

$$
F_{(1,58)}=\frac{S S E_{\text {red }}-S S E_{\text {full }} / d_{\text {full }}-d_{\text {red }}}{S S E_{\text {full }} /\left(n-d_{\text {full }}-1\right)} .
$$

Here SSE is the sum of squared errors, $d$ is the number of covariates in the full or reduced model, and $n$ is the number of participants.

\section{Results}

We did not detect significant associations between IHTT and FA, perhaps because the group covariate included in the analysis explained most of the variance. During a preliminary review of the distribution of IHTT scores of the TBI participants and the healthy control subjects, we observed that the distribution of scores in the TBI group was highly skewed. A cutoff score of $18 \mathrm{~ms}$ optimized the balance between sensitivity and specificity, resulting in only 3 of 31 healthy control subjects having IHTTs within the slow range. Half of the TBI group $(n=16)$ had IHTT scores within 1.5 SDs of the normal range, or $<18$ ms. The balance of the TBI group had very slow IHTTs, $>18 \mathrm{~ms}$. The normal range was defined based on the IHTT scores for the healthy control group, and a cutting score was chosen to maximize sensitivity and specificity. Consequently, the TBI group was split into the following two subgroups: normal IHTT TBI $(n=16)$ and slow IHTT TBI $(n=16)$ children (Table 2). The IHTT-slow and IHTT-normal groups did not differ significantly in time since injury, injury severity, as assessed by GCS ( $p=0.40)$ or the presence of any of nine CT scan findings (increased intracranial pressure, diffuse axonal injury, subarachnoid hemorrhage, ventricular hemorrhage, epidural hematoma, subdural hematoma, intracerebral hematoma, contusions, and skull fracture; all $p>0.16$ ). Most of these lesions are cortical, but one participant had a small detectable lesion in the CC, an intracerebral hematoma. This participant was in the IHTT-normal group, and when we compared the tractography in this participant to that in other TBI participants, it appeared that the lesion had not biased the reconstruction of the fibers traveling through the CC.

\section{IHTT-slow versus control}

When we compared the IHTT-slow TBI subgroup to the control group, we found significant differences in FA across 17 of the 18 tracts tested (all but $c_{\text {c }} \_$l; critical $p=0.0038$ ). We also found significant differences in MD across 13 of the 18 tracts tested (all but atr_l, cgc_r, cst_l, cst_r, and fornix; critical $p=0.0036$ ). For all of these, FA was higher in the control group and MD was lower. These are shown in Table 3 and Figures 1 and 2.

\section{IHTT-normal versus control}

We compared the IHTT-normal TBI subgroup to the control group, and we found significant differences in FA across the following four tracts: CC-frontal, cst_l, cst_r, and ifo_l (critical $p=$ $\left.6.5 \times 10^{-4}\right)$. All of these clusters were areas of higher FA in the control group. There were no significant differences in MD. These are shown in Table 3 and Figure 3.

\section{Cognitive performance}

We also tested our groups for differences in cognitive performance. A one-way ANOVA revealed significant group differ- 
ences among the IHTT-slow, IHTTnormal, and control groups in performance index $\left(F_{(2,60)}=6.68 ; p=\right.$ 0.003 ) (Table 4). Our element-wise regression testing for associations between $\mathrm{FA}$ or $\mathrm{MD}$ and cognitive performance yielded significant results only in the FA of the CC-frontal tract (critical $p=4.42 \times$ $10^{-4}$; all others were $<1 \%$ of the tract). Our partial $F$ test, examining the joint effect of IHTT and FA on cognitive performance, was broadly significant across all tracts. This is shown in Table 5 and Figure 4. Across all of these points, the addition of element-wise FA to the model explained significantly more variance in cognitive performance than the reduced model including only age, sex, and IHTT group.

\section{Discussion}

In this study, we combined advanced tract-based analyses with an assessment of callosal function to examine outcome heterogeneity within TBI. Of the participants in our TBI group, 16 had an IHTT that did not deviate significantly from the control group. Sixteen TBI participants had IHTTs that deviated significantly, by at least 1.5 SDs, based on the healthy control data. Based on this bimodal distribution, we investigated and confirmed our initial hypotheses that these differences in callosal function were also accompanied by differences in callosal integrity as well as differences in cognitive performance.

When we compared the IHTT-slow

TBI group to the healthy control group, we found significant differences in callosal WM integrity, as well as the integrity of the association and projection tract systems tested. Lower FA and higher MD in the IHTT-slow group suggest myelin disruption (Klawiter et al., 2011; Thomason and Thompson, 2011). When we compared the IHTT-normal TBI group to the healthy control group, we found only a few areas where the TBI group had significantly lower FA and no significant differences in MD. Also, there were no detectable differences in the posterior callosal tracts, the region targeted by the visual task completed during the ERP sequence. This relative sparing of WM integrity is most likely a significant contributor to this group having normal IHTTs. It is well known that demyelination of axons increases conduction time and impairs the integrity of the signal (Waxman, 1977). As a relatively direct measure (i.e., high temporal resolution) of CC functional integrity, ERP-measured IHTT is a very useful complement to HARDI and other sensitive MRI measures used to assess the structural integrity of the CC. Practically, EEG is less expensive than MRI and has fewer exclusionary criteria (e.g., braces in children). Our data, along with prior studies of IHTT or DWI separately, support the theory that TBI can cause extensive and prolonged damage to myelin integrity, impairing the function of those tracts.

This is the first study, to our knowledge, to examine the relationship between IHTT and DWI in TBI. We studied children in the post-acute phase (1-5 months) of injury after msTBI. During this post-acute phase, there is a divergence within the msTBI group that has a bearing on postinjury cognitive outcome. We found that the IHTT-slow group had significantly poorer performance than the IHTT-normal group (8.5\% reduction in average performance), whose performance was slightly poorer than that of the healthy control group (healthy control subjects performed $4.0 \%$ better on average). We also found an association between cognitive performance and WM integrity, with better performance associated with higher FA in the CC-frontal and arc_l tracts. These results overlap with areas where we found differences in WM integrity between the IHTT-slow and control groups. In prior studies, the posterior CC was particularly vulnerable to TBI and was atrophied postinjury in some cases (Lindenberg et al., 1955; Gentry et al., 1988; Mendelsohn et al., 1992; Vuilleumier and Assal, 1995; Benavidez et al., 1999; Peru et al., 2003; Shiramizu et al., 2008; Slawik et al., 2009). Lesions in the CC post-TBI may result from TAI, arrested development in the CC, or secondary injuries (Shiramizu et al., 2008). Slawik et al. (2009) observed that the anterior CC was also vulnerable to secondary injury following TBI. Our study demonstrated a loss of CC integrity in both the posterior and anterior regions in the subset of children with TBI who had impaired IHTT; that is, who demonstrated slower CC functioning than control subjects and the normal IHTT TBI subgroup.

We additionally demonstrated that these deficits in CC structural and functional integrity impact cognitive performance. 


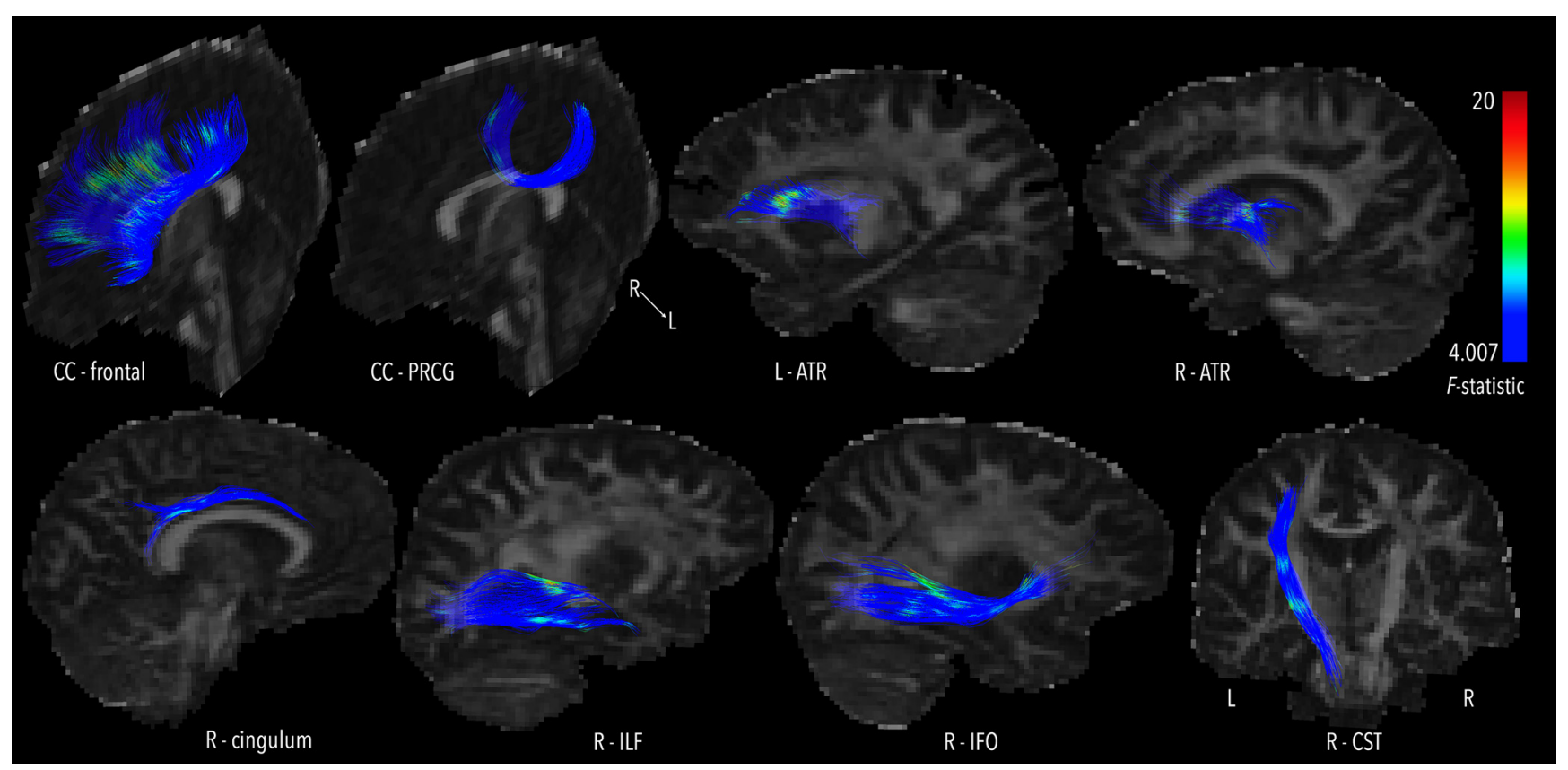

Figure 5. Joint effects of FA and IHTT on cognitive performance. Significant results from the partial $F$ test comparing a reduced model (age, sex, and IHTT group) with a full model (age, sex, IHTT-group, and element-wise FA) with cognitive performance as the response variable. For simplicity, only tracts with at least $5 \%$ of the tract significant are shown. The $F$ statistic is shown along the tract corresponding to the color bar. PRCG, Precentral gyrus; ATR, anterior thalamic radiation; ILF, inferior longitudinal fasciculus; IFOF, inferior fronto-occipital fasciculus; CST, corticospinal tract.

Lower FA, across the brain, and slower IHTT were associated with significantly poorer performance index. Our element-wise test of FA and cognitive performance had limited results (Fig. 4). There was a significant group effect on cognitive performance, with the IHTT-slow group performing more poorly than the IHTT-normal group, who performed more poorly than the healthy control group. However, our partial $F$ test revealed that we could explain significantly more of the variance in cognitive performance by adding element-wise FA to the model including the IHTT group, as shown in Figure 5. This figure displays which parts of which tracts bolstered the prediction of cognitive performance, when added to the model including age, sex, and IHTT group. Our results indicate to us that the structural and functional integrity of the $\mathrm{CC}$ together explain more of the variance in cognitive performance than functional or structural information alone.

Multimodal studies such as ours play an important role in validating and supporting existing interpretations of results. From animal studies, there is broad support for the interpretation that decreases in FA and increases in MD can indicate demyelination (Song et al., 2005), but multimodal in vivo data are generally more convincing. Here, decreased FA and increased MD were associated with increased IHTT, our measure of CC function, which lends further support to the interpretation that this combination of results suggests demyelination. This has implications beyond the field of TBI. Other biological interpretations that often are suggested to explain decreases in FA or increases in MD are decreased axonal packing, edema, and inflammation. While edema generally peaks in a matter of days postinjury before subsiding (Marmarou, 2007), inflammation may still persist months later; this time course is not well characterized in humans. Our recent study examining volumetric differences between TBI and control subjects cross-sectionally found CSF expansion in the lateral ventricles, likely at the expense of callosal and subcortical tissue. We found possible evidence of persistent inflammation, in the form of significant volume expansion in the
TBI group, not explained by CSF expansion, in the parietal WM, not in the callosal tracts found here. We are beginning a protocol to integrate our HARDI data with our MRS data. MRS yields spectra of neural metabolites that reflect neural integrity, inflammation, and energy metabolism. These data will help us understand our results in greater depth. For this analysis, only HARDI was used to extract tract-based white matter integrity. We did not collect susceptibility-weighted imaging or T2-fluid-attenuated inversion recovery, which might have offered greater information about microhemorrhages and diffuse axonal injury.

TBI can lead to decreases in FA and can cause difficulties in tracking fibers in diffusion images in TBI (Xu et al., 2007). Interruptions in the normal diffusion MRI signal can cause problems for some tractography methods because more severely disrupted areas are skipped in analysis. For deterministic methods that rely on seeking a fiber path by looking only at the dominant direction of diffusion from one voxel to the next, this may bias results or give an incomplete picture. As with other methods, diffusivity measures were assessed along the fibers, but, unlike other methods, our group comparisons are not constrained to where fibers were reconstructed. In an area of low FA, where a fiber might be dropped, the FA at the point in the registered FA map is used for group comparison. Diffusivity measures at the point are interpolated to subvoxel resolution. The fiber maps are used to generate standardized spaces for group comparisons and allow us to reference our results to tract A versus tract B, even though the group comparisons do not depend on the accurate assignment of voxels to the correct fiber tracts. All subjects are compared in the space of one subject's fiber coordinates, after registering each subject's FA map to that example subject's FA.

Heterogeneity in postinjury outcomes is an important issue in TBI and is still poorly understood. Multimodal studies such as ours are critical to understanding and tracking this heterogeneity. Our study shows that postinjury heterogeneity is explained in part by data from EEG- and DWI-derived measures. This indicates that these will be reliable biomarkers for tracking outcome. 
Some studies of pediatric TBI report a normal developmental trajectory of the CC between 3 months and 3 years postinjury (Levin et al., 2000), while others report mixed results, with some indicators of recovery (a modest increase in FA longitudinally) and others of continued degeneration (volume decreases; Wu et al., 2010). Our prior cross-sectional study examining this group at two time points found more extensive group differences at the chronic time point (13-19 months postinjury) than in the postacute phase. This is likely due in part to the within-group heterogeneity in the post-acute phase. Local disruption appears to dominate and differs spatially between participants, but over the first year postinjury this disruption may spread and become global (Dennis et al., 2015). Longitudinal analyses will shed more light on this progression. Our current results indicate a divergence, already present in the post-acute phase, which has important implications for how future studies are approached and interpreted.

\section{References}

Andreassi JL, Okamura H, Stern M (1975) Hemispheric asymmetries in the visual cortical evoked potential as a function of stimulus location. Psychophysiology 12:541-546. CrossRef Medline

Avants BB, Tustison NJ, Song G, Cook PA, Klein A, Gee JC (2011) A reproducible evaluation of ANTs similarity metric performance in brain image registration. Neuroimage 54:2033-2044. CrossRef Medline

Babikian T, Asarnow R (2009) Neurocognitive outcomes and recovery after pediatric TBI: meta-analytic review of the literature. Neuropsychology 23:283-296. CrossRef Medline

Babikian T, Marion SD, Copeland S, Alger JR, O’Neill J, Cazalis F, Mink R, Giza CC, Vu JA, Hilleary SM, Kernan CL, Newman N, Asarnow RF (2010) Metabolic levels in the corpus callosum and their structural and behavioral correlates after moderate to severe pediatric TBI. J Neurotrauma 27:473-481. CrossRef Medline

Bartzokis G, Lu PH, Tingus K, Mendez MF, Richard A, Peters DG, Oluwadara B, Barrall KA, Finn JP, Villablanca P, Thompson PM, Mintz J (2010) Lifespan trajectory of myelin integrity and maximum motor speed. Neurobiol Aging 31:1554-1562. CrossRef Medline

Benavidez DA, Fletcher JM, Hannay HJ, Bland ST, Caudle SE, Mendelsohn DB, Yeakley J, Brunder DG, Harward H, Song J, Perachio NA, Bruce D, Scheibel RS, Lilly MA, Verger-Maestre K, Levin HS (1999) Corpus callosum damage and interhemispheric transfer of information following closed head injury in children. Cortex 35:315-336. CrossRef Medline

Benjamini Y, Hochberg Y (1995) Controlling the false discovery rate: a practical and powerful approach to multiple testing. J R Stat Soc Series B Stat Methodol 57:289-300.

Brown WS, Jeeves MA (1993) Bilateral visual field processing and evoked potential interhemispheric transmission time. Neuropsychologia 31: 1267-1281. CrossRef Medline

Brown WS, Jeeves MA, Dietrich R, Burnison DS (1999) Bilateral field advantage and evoked potential interhemispheric transmission in commissurotomy and callosal agenesis. Neuropsychologia 37:1165-1180. CrossRef Medline

Büki A, Povlishock JT (2006) All roads lead to disconnection? Traumatic axonal injury revisited. Acta neurochirurgica 148:181-194. CrossRef Medline

Caeyenberghs K, Leemans A, Coxon J, Leunissen I, Drijkoningen D, Geurts M, Gooijers J, Michiels K, Sunaert S, Swinnen SP (2011) Bimanual coordination and corpus callosum microstructure in young adults with traumatic brain injury: a diffusion tensor imaging study. J Neurotrauma 28:897-913. CrossRef Medline

Catani M, Allin MP, Husain M, Pugliese L, Mesulam MM, Murray RM, Jones DK (2007) Symmetries in human brain language pathways correlate with verbal recall. Proc Natl Acad Sci U S A 104:17163-17168. CrossRef Medline

Damasio AR, Chui HC, Corbett J, Kassel N (1980) Posterior callosal section in a non-epileptic patient. J Neurol Neurosurg Psychiatry 43:351-356. CrossRef Medline

Delis DC, Kramer JH, Kaplan E, Ober A (1994) California verbal learning test-children's version (CVLT-C) manual. San Antonio, TX: Psychological Corporation.
Delis DC, Kaplan E, Kramer JH (2001) Delis-Kaplan executive function system (D-KEFS). San Antonio, TX: Psychological Corporation.

Dennis EL, Jin Y, Villalon-Reina JE, Zhan L, Kernan CL, Babikian T, Mink RB, Babbitt CJ, Johnson JL, Giza CC, Thompson PM, Asarnow RF (2015) White matter disruption in moderate/severe pediatric traumatic brain injury: advanced tract-based analyses. Neuroimage Clin 7:493-505. CrossRef Medline

Ellis MU, Deboard Marion S, McArthur D, Babikian T, Giza CC, Kernan CL, Newman N, Moran L, Akarakian R, Houshiarnejad A, Mink R, Johnson J, Babbitt C, Olsen A, Asarnow RF (2015) The UCLA study of children with moderate to severe traumatic brain injury: event-related potential measure of interhemispheric transfer time. J Neurotrauma, in press.

Ewing-Cobbs L, Prasad MR, Kramer L, Cox CS Jr, Baumgartner J, Fletcher S, Mendez D, Barnes M, Zhang X, Swank P (2006) Late intellectual and academic outcomes following traumatic brain injury sustained during early childhood. J Neurosurg 105 [4 Suppl]:287-296. Medline

Ewing-Cobbs L, Prasad MR, Swank P, Kramer L, Cox CS Jr, Fletcher JM, Barnes M, Zhang X, Hasan KM (2008) Arrested development and disrupted callosal microstructure following pediatric traumatic brain injury: relation to neurobehavioral outcomes. Neuroimage 42:1305-1315. CrossRef Medline

Gazzaniga MS, Freedman H (1973) Observations on visual processes after posterior callosal section. Neurology 23:1126-1130. CrossRef Medline

Gentry LR, Thompson B, Godersky JC (1988) Trauma to the corpus callosum: MR features. AJNR Am J Neuroradiol 9:1129-1138. Medline

Hulkower MB, Poliak DB, Rosenbaum SB, Zimmerman ME, Lipton ML (2013) A decade of DTI in traumatic brain injury: 10 years and 100 articles later. AJNR Am J Neuroradiol 34:2064-2074. CrossRef Medline

Jin Y, Shi Y, Jahanshad N, Aganj I, Sapiro G, Toga AW, Thompson PM (2011) 3D elastic registration improves HARDI-derived fiber alignment and automated tract clustering. Paper presented at 2011 IEEE International Symposium on Biomedical Imaging: From Nano to Macro, Chicago, IL, March. CrossRef

Jin Y, Shi Y, Zhan L, Li J, De Zubicaray GI, McMahon KL, Martin NG, Wright MJ, Thompson PM (2012) Automatic population HARDI white matter tract clustering by label fusion of multiple tract atlases. Multimodal Brain Image Anal Lecture Notes Comput Sci 7509:147-156. CrossRef

Jin Y, Shi Y, Zhan L, de Zubicaray GI, McMahon KL, Martin NG, Wright MJ, Thompson PM (2013) Labeling white matter tracts in HARDI by fusing multiple tract atlases with applications to genetics. Paper presented at 2013 IEEE 10th International Symposium on Biomedical Imaging: From Nano to Macro, San Francisco, CA, April.

Jin Y, Shi Y, Zhan L, Gutman BA, de Zubicaray GI, McMahon KL, Wright MJ, Toga AW, Thompson PM (2014) Automatic clustering of white matter fibers in brain diffusion MRI with an application to genetics. Neuroimage 100:75-90. CrossRef Medline

Klawiter EC, Schmidt RE, Trinkaus K, Liang HF, Budde MD, Naismith RT, Song SK, Cross AH, Benzinger TL (2011) Radial diffusivity predicts demyelination in ex vivo multiple sclerosis spinal cords. Neuroimage 55: 1454-1460. CrossRef Medline

Kochunov P, Williamson DE, Lancaster J, Fox P, Cornell J, Blangero J, Glahn DC (2012) Fractional anisotropy of water diffusion in cerebral white matter across the lifespan. Neurobiol Aging 33:9-20. CrossRef Medline

Larson EB, Brown WS (1997) Bilateral field interactions, hemispheric specialization and evoked potential interhemispheric transmission time. Neuropsychologia 35:573-581. CrossRef Medline

Lassonde M, Sauerwein H, McCabe N, Laurencelle L, Geoffroy G (1988) Extent and limits of cerebral adjustment to early section or congenital absence of the corpus callosum. Behav Brain Res 30:165-181. CrossRef Medline

Ledlow A, Swanson JM, Kinsbourne M (1978) Differences in reaction times and average evoked potentials as a function of direct and indirect neural pathways. Ann Neurol 3:525-530. CrossRef Medline

Levin HS, Benavidez DA, Verger-Maestre K, Perachio N, Song J, Mendelsohn DB, Fletcher JM (2000) Reduction of corpus callosum growth after severe traumatic brain injury in children. Neurology 54:647-653. CrossRef Medline

Lindenberg R, Fisher RS, Durlacher SH, Lovitt WV Jr, Freytag E (1955) Lesions of the corpus callosum following blunt mechanical trauma to the head. J Pathol 31:297.

Marmarou A (2007) A review of progress in understanding the pathophysiology and treatment of brain edema. Neurosurg Focus 22:E1. Medline 
Mathias JL, Bigler ED, Jones NR, Bowden SC, Barrett-Woodbridge M, Brown GC, Taylor DJ (2004) Neuropsychological and information processing performance and its relationship to white matter changes following moderate and severe traumatic brain injury: a preliminary study. Appl Neuropsychol 11:134-152. CrossRef Medline

Mendelsohn DB, Levin HS, Harward H, Bruce D (1992) Corpus callosum lesions after closed head injury in children: MRI, clinical features and outcome. Neuroradiology 34:384-388. CrossRef Medline

Nagy Z, Westerberg H, Klingberg T (2004) Maturation of white matter is associated with the development of cognitive functions during childhood. J Cogn Neurosci 16:1227-1233. CrossRef Medline

Peru A, Beltramello A, Moro V, Sattibaldi L, Berlucchi G (2003) Temporary and permanent signs of interhemispheric disconnection after traumatic brain injury. Neuropsychologia 41:634-643. CrossRef Medline

Rugg MD, Milner AD, Lines CR (1985) Visual evoked potentials to lateralised stimuli in two cases of callosal agenesis. J Neurol Neurosurg Psychiatry 48:367-373. CrossRef Medline

Saron CD, Davidson RJ (1989) Visual evoked potential measures of interhemispheric transfer time in humans. Behav Neurosci 103:1115-1138. CrossRef Medline

Sauerwein H, Lassonde MC (1983) Intra-and interhemispheric processing of visual information in callosal agenesis. Neuropsychologia 21:167-171. CrossRef Medline

Schulte T, Sullivan EV, Müller-Oehring EM, Adalsteinsson E, Pfefferbaum A (2005) Corpus callosal microstructural integrity influences interhemispheric processing: a diffusion tensor imaging study. Cereb Cortex 15: 1384-1392. CrossRef Medline

Shiramizu H, Masuko A, Ishizaka H, Shibata M, Atsumi H, Imai M, Osada T, Mizokami Y, Baba T, Matsumae M (2008) Mechanism of injury to the corpus callosum, with particular reference to the anatomical relationship between site of injury and adjacent brain structures. Neurol Med Chir (Tokyo) 48:1-7. CrossRef Medline

Slawik H, Salmond CH, Taylor-Tavares JV, Williams GB, Sahakian BJ, Tasker RC (2009) Frontal cerebral vulnerability and executive deficits from raised intracranial pressure in child traumatic brain injury. J Neurotrauma 26:1891-1903. CrossRef Medline
Song SK, Yoshino J, Le TQ, Lin SJ, Sun SW, Cross AH, Armstrong RC (2005) Demyelination increases radial diffusivity in corpus callosum of mouse brain. Neuroimage 26:132-140. CrossRef Medline

Sugishita M, Yoshioka M (1987) Visual processes in a hemialexic patient with posterior callosal section. Neuropsychologia 25:329-339. CrossRef Medline

Thomason ME, Thompson PM (2011) Diffusion imaging, white matter, and psychopathology. Annu Rev Clin Psychol 7:63-85. CrossRef Medline

Vuilleumier P, Assal G (1995) Complete callosal disconnection after closed head injury. Clin Neurol Neurosurg 97:39-46. CrossRef Medline

Waxman SG (1977) Conduction in myelinated, unmyelinated, and demyelinated fibers. Arch Neurol 34:585-589. CrossRef Medline

Wechsler D (2003) Wechsler intelligence scale for children, Ed 4 (WISCIV). San Antonio, TX: Psychological Corporation.

Westerhausen R, Kreuder F, Woerner W, Huster RJ, Smit CM, Schweiger E, Wittling W (2006) Interhemispheric transfer time and structural properties of the corpus callosum. Neurosci Lett 409:140-145. CrossRef Medline

Whitford TJ, Kubicki M, Ghorashi S, Schneiderman JS, Hawley KJ, McCarley RW, Shenton ME, Spencer KM (2011) Predicting inter-hemispheric transfer time from the diffusion properties of the corpus callosum in healthy individuals and schizophrenia patients: a combined ERP and DTI study. Neuroimage 54:2318-2329. CrossRef Medline

Wu TC, Wilde EA, Bigler ED, Li X, Merkley TL, Yallampalli R, McCauley SR, Schnelle KP, Vasquez AC, Chu Z, Hanten G, Hunter JV, Levin HS (2010) Longitudinal changes in the corpus callosum following pediatric traumatic brain injury. Dev Neurosci 32:361-373. CrossRef Medline

Xu J, Rasmussen IA, Lagopoulos J, Håberg A (2007) Diffuse axonal injury in severe traumatic brain injury visualized using high-resolution diffusion tensor imaging. J Neurotrauma 24:753-765. CrossRef Medline

Zhang Y, Zhang J, Oishi K, Faria AV, Jiang H, Li X, Akhter K, Rosa-Neto P, Pike GB, Evans A, Toga AW, Woods R, Mazziotta JC, Miller MI, van Zij PC, Mori S (2010) Atlas-guided tract reconstruction for automated and comprehensive examination of the white matter anatomy. Neuroimage 52:1289-1301. CrossRef Medline 